\title{
Probe-Based Confocal Laser Endomicroscopy and Barrett's Esophagus: Just a Scientific Toy or Significant Improvement in Diagnosis?
}

\author{
Luka Vranića Tin Nadarevićb Davor Štimac ${ }^{a}$ \\ aDepartment of Gastroenterology, Clinical Hospital Centre Rijeka, Faculty of Medicine, University of Rijeka, \\ Rijeka, Croatia; 'bepartment of Radiology, Clinical Hospital Centre Rijeka, Faculty of Medicine, University of \\ Rijeka, Rijeka, Croatia
}

\section{Keywords \\ Confocal laser endomicroscopy · Barrett's esophagus . \\ Dysplasia · Early esophageal carcinoma}

\begin{abstract}
Background: Barrett's esophagus (BE) requires surveillance to identify potential neoplasia at an early stage. The standard surveillance regimen includes random 4-quadrant biopsies by Seattle protocol. Main limitations of random biopsies are high risk of sampling error, difficulties in histology interpretation, common inadequate classification of pathohistological changes, increased risk of bleeding, and time necessary to acquire the final diagnosis. Probe-based confocal laser endomicroscopy ( $p C L E$ ) has emerged as a potential tool with an aim to overcome these obvious limitations. Summary: pCLE represents a real-time microscopic imaging method that offers evaluation of epithelial and subepithelial structures with 1,000-fold magnification. In theory, pCLE has potential to eliminate the need for biopsy in BE patients. The main advantages would be real-time diagnosis and decision-making, greater diagnostic accuracy, and evaluation of larger area compared to random biopsies. Clinical pCLE studies in the esophagus show high diagnostic
\end{abstract}

karger@karger.com

(c) 2021 S. Karger AG, Basel

www.karger.com/dd

Karger" accuracy, and its high negative predictive value offers high reliability and confidence to exclude dysplastic and neoplastic lesions. However, it still cannot replace histopathology due to lower positive predictive value and sensitivity. Key Messages: Despite promising results, its role in routine use in patients with $B E$ remains questionable primarily due to lack of well-organized double-blind randomized trials.

(c) 2021 S. Karger AG, Basel

\section{Introduction}

Barrett's esophagus (BE) represents replacement of the stratified squamous epithelium of the lower esophagus with the metaplastic columnar glandular epithelium. It is known as a predominant risk factor for esophageal adenocarcinoma (EA), and therefore it requires clinical surveillance in order to identify potential neoplasia at early stage. Its pathohistological features include intestinal metaplasia (IM), low-grade dysplasia (LGD), high-grade dysplasia (HGD), and EA [1]. Standard surveillance regimens to identify early neoplasia, recommended by the European Society of Gastrointestinal Endoscopy (ESGE), include periodical upper endoscopy with random biop- 
sies, usually taken by Seattle protocol (random 4-quadrant biopsies taken every $2 \mathrm{~cm}$ within the Barrett's segment, starting from the upper end of the gastric folds) and biopsies of visible lesions [2]. However, current standard surveillance has low diagnostic yield since dysplastic lesions are difficult or sometimes impossible to identify and due to sampling error of random biopsies. Therefore, interest and need of new endoscopic imaging techniques has been generated. Confocal laser endomicroscopy (CLE) represents real-time microscopic imaging during endoscopic examination. A 1,000-fold magnification enables visualization of cells, vascular patterns, and glandular architecture of a certain point of interest within the tissue [3]. Until today, CLE has been evaluated in multiple studies in various locations in the digestive tract, but its clinical usefulness is still questionable.

\section{CLE as a Diagnostic Method}

In the last 15 years, CLE has emerged as a potential tool that would make gastroenterologists' and pathologists' diagnostic arsenal wider with the aim to overcome obvious limitations of random biopsy sampling techniques allowing to optimize and rationalize the management of $\mathrm{BE}$ [4]. Currently, 2 CLE systems exist: an endoscope-based endomicroscopy (eCLE) and probe-based endomicroscopy (pCLE). In the eCLE system (Pentax Medical Corporation, Tokyo, Japan), the confocal imaging system is incorporated to the tip of the high-resolution endoscope allowing examinations of only certain parts of the digestive tract. This system is not commercially available, despite some promising research results $[3,4]$. The pCLE system was developed by Mauna Kea Technologies (Cellvizio; Paris, France) and represents a newer-generation probe that can be inserted in a working channel of a standard endoscope and can be used to examine the entire gastrointestinal tract. Until today, pCLE has been used, mostly through research, in the esophagus, stomach, and intestine, as well as in the biliary tract (during cholangioscopy) and pancreas (in the main pancreatic duct with cholangioscopy and to conduct real-time examination of pancreatic masses or cysts during endoscopic ultrasound). Moreover, pCLE has been utilized in pulmonology, urology, neurosurgery, and other medical fields [3].

CLE is based upon the principle of tissue illumination with low-power laser and detection of the reflecting fluorescent light from the tissue. The term "confocal" refers to the fact that both illumination and detection systems are in the same focal plane [5]. In the pCLE system, the light source and laser scanning unit are integrated in an external unit, meaning that a miniprobe serves as a passive conduit. These are inserted in working channels of endoscopes and direct low-power laser, emitted from the external unit to the mucosal point of interest through optical fibers and lenses at their tip. The laser light is directed to a selected depth, depending on examining tissue, and then reflected fluorescent light is refocused to the detection system by the same lens at the tip [3-5].

With the resolution up to 1 micron, this technology enables real-time imaging of certain epithelial and subepithelial structures at the cellular level. However, it provides a static imaging depth of 55-65 $\mu \mathrm{m}$, unlike eCLE, without the possibility of invasion depth assessment [1].

Since tissue has natural limited fluorescence, to detect laser reflections of the tissue of interest and to generate an image, a contrast agent is required for CLE. Most studies have used fluorescein, a substance approved by the Food and Drug Administration (FDA) for retinal angiography. The doses between studies vary, but most have used 2.5 $\mathrm{mL}$ of $10 \%$ of fluorescein sodium or $0.1 \mathrm{~mL} / \mathrm{kg}$ of $1 \% \mathrm{flu}-$ orescein sodium intravenously $[1,3]$. Safety of fluorescein was examined in 2,272 patients at 16 different centers. There were no serious adverse events, and only mild events such as nausea, vomiting, transient hypotension, mild epigastric pain, or rash were reported in $1.4 \%$ of patients [6].

Among different miniprobes, GastroFlex ${ }^{\mathrm{TM}} \mathrm{UHD}$ has been used for esophagus examination. Examination should start, as usually, with high-definition white light endoscopy (HD-WLE) examination combined with chromoendoscopy or narrow-band imaging (NBI) to define areas of interest. Before endomicroscopy exam, sodium fluorescein is applied. The miniprobe is inserted through the working channel and pointed with the endoscope to the areas of interest to perform real-time examination and record videos for postprocedural analysis. According to the manufacturer, a miniprobe can be used up to 20 times after proper disinfection [3].

\section{Current BE Surveillance - Is It Enough?}

According to the latest ESGE guidelines, high-definition endoscopy is highly recommended to use for BE surveillance, while the routine use of advanced endoscopy imaging, including CLE, is not recommended. Moreover, endoscopic random biopsies by Seattle protocol and biopsies of the visible mucosal abnormalities are still considered as a gold standard for diagnosing BE, LGD, HGD, 
Table 1. Comparison of standard biopsies versus pCLE characteristics

\begin{tabular}{lll}
\hline Parameters & $\begin{array}{l}\text { Four-quadrant biopsies } \\
\text { (Seattle protocol) }\end{array}$ & pCLE \\
\hline Microscopic imaging & + & + \\
Histopathological diagnosis & + & - \\
Depth assessment & + & - \\
Type of imaging & Static & Dynamic \\
Examined area & Small & Large \\
Evaluated by & Pathologist & Real-time - gastroenterologist; blinded review - gastroenterologist/ \\
& Prolonged & pathologist \\
Diagnose timing & - & Real-time/prolonged (postprocedural image analysis) \\
Possibility of guiding endoscopic procedure & Low & High \\
Costs & & \\
\hline
\end{tabular}

pCLE, probe-based confocal laser endomicroscopy.

EA, and squamous cell carcinoma [2]. However, there have been several limitations regarding the standard recommended diagnostic practice of periodic surveillance mucosal biopsies. First, it is not surprising that many endoscopists do not adhere to Seattle protocol in their daily practice because it can be inconvenient. For example, during the examination of a $10-\mathrm{cm}$ long BE, 20 overall biopsies should be taken, according to current guidelines. This approach can be challenging for the endoscopist and intolerable for the patient, especially in centers where sedation is not routinely practiced, not to mention time consuming and technically difficult. Abrams et al. [7] revealed that in overall 2,245 $\mathrm{BE}$ surveillance endoscopies, in only $51 \%$ of cases adherence to biopsy guidelines was practiced. Adherence was inversely related to length of $\mathrm{BE}$, meaning that in only $17.9 \%$ of $6-8 \mathrm{~cm}$ long $\mathrm{BE}$ and in $10.9 \%$ cases of $>9 \mathrm{~cm}$ long BE, biopsies were performed by the guidelines protocol. More importantly, nonadherence resulted in lower dysplasia detection rate. Second, the diagnostic accuracy of esophageal biopsies is not that high to be completely reliable, especially in patients with LGD. This is partially due to randomly taken biopsies that assess only a small surface of BE and partially due to overdiagnosing or underdiagnosing by pathologists. In a study done by Cameron et al. [8], distribution and extent of dysplasia and esophageal carcinoma were examined by histology mapping on resected esophageal specimens due to HGD or EA. Median BE surface was $32 \mathrm{~cm}^{2}$, with HGD and EA 1.3 and $1.1 \mathrm{~cm}^{2}$, respectively, meaning that early carcinoma and high-risk lesions are extremely focal and easy to miss during biopsy protocol $[8,9]$. On the other hand, LGD is frequently being overdiagnosed by pathologists. It is often due to "overestimation of regenerative changes" that can be very similar to dysplasia under the microscope. In a retrospective cohort study, after expert pathologist's reassessment of initially diagnosed LGD within BE, $73 \%$ of LGD was downgraded to be nondysplastic BE or indefinite for dysplasia [10]. This misdiagnosis could have negative impact on patients, since LGD is a lesion with increased risk of progression to advanced neoplasia. Also, false-positive LGD diagnosis could lead to unnecessary therapeutic procedures and accompanied procedural risks.

The abovementioned limitations of standard care enhance the need of new diagnostic approaches that could either be added to or completely replace 4 -quadrant biopsies. These approaches, including pCLE, are currently in development and are being tested to improve the detection rate and the diagnostic accuracy in neoplasia detection in patients with $\mathrm{BE}$. Comparison of characteristics of $\mathrm{pCLE}$ and the standard biopsy procedure is presented in Table 1.

\section{CLE for the Diagnosis of BE and Related Neoplasia}

\section{Detection of IM}

During the last 15 years, pCLE has been studied extensively in not only different sites of the gastrointestinal tract but also in many other organs. In the esophagus, pCLE could be used to detect IM and to establish the diagnosis of $\mathrm{BE}$. Until today, the diagnosis of $\mathrm{BE}$ is primarily a histopathological diagnosis which is made if the columnar epithelium is visually observed with a minimum length of $1 \mathrm{~cm}$ above the end of gastric folds and specialized IM present at histopathological examination [2]. 


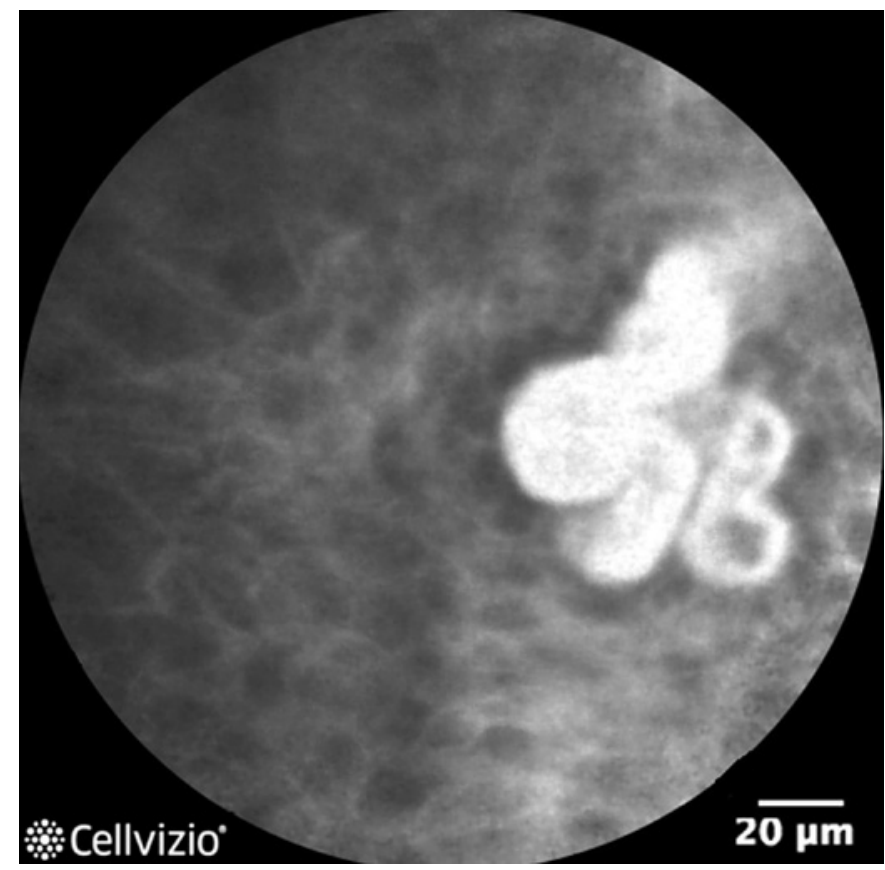

Fig. 1. Normal squamous epithelium: flat and scale-like cells with intrapapillary loops (credit: Mauna Kea Technologies).

However, the technique such as $\mathrm{pCLE}$, which provides "in vivo" examination at cellular level, could in the future be simplified to establish the diagnosis. The Mainz Confocal Barrett's Classification uses cellular and vascular architecture to differentiate IM from the normal cardiac epithelium both in superficial and deep parts of mucosa. Normal gastric mucosa has a typical cobblestone appearance, round and regular glands (gastric pits) with regularshaped capillaries in deeper mucosa. Normal squamous epithelium is characterized by flat and scale-like cells with intrapapillary loops (shown in Fig. 1). On the other hand, nondysplastic BE (IM) on CLE is defined by the presence of goblet cells within columnar mucosa that produce dark mucin and regular-shaped capillaries in the upper and deep mucosa (shown in Fig. 2) [1,3,11]. A pilot study of CLE by Kiesslich et al. [11] proposed the abovementioned classification which yielded a sensitivity, specificity, and diagnostic accuracy of 98,94 , and $97 \%$, respectively, in comparison with histologic findings. Positive predictive value (PPV) was 97\%, and negative predictive value (NPV) was $96 \%$ with the mean kappa value for interobserver and intraobserver agreement of 0.843 and 0.892 , respectively. Following the proposal of these criteria, a new Miami classification was proposed in 2011 for pCLE which was similar to Mainz classification. Normal colum-

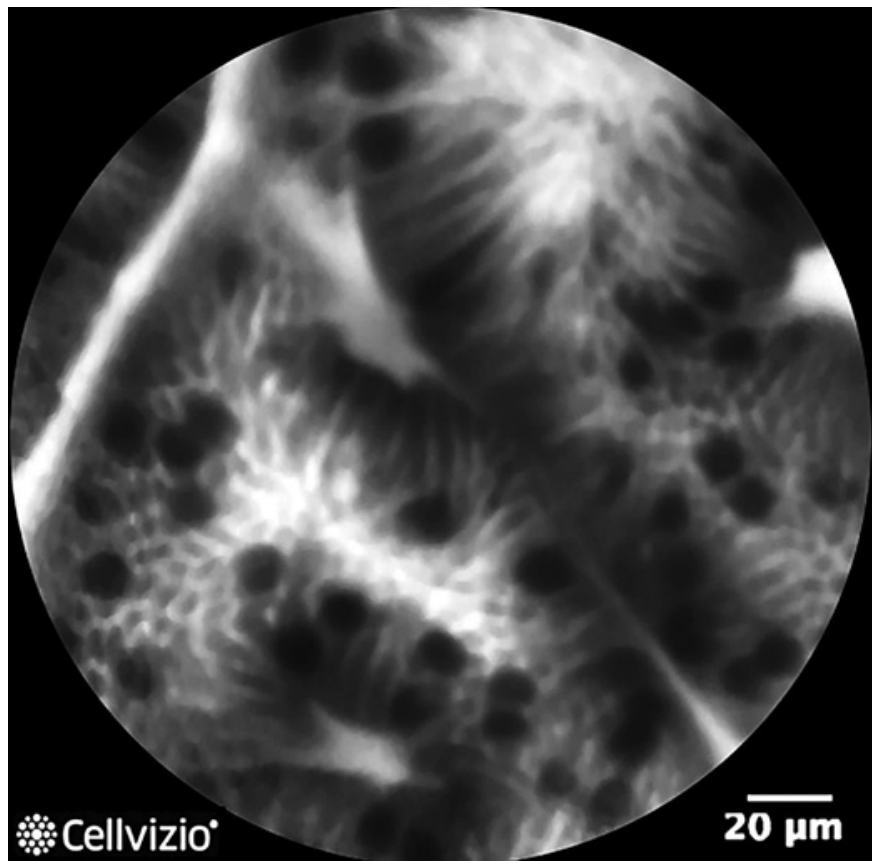

Fig. 2. IM: regular columnar-lined epithelium and dark mucin in goblet cells (credit: Mauna Kea Technologies). IM, intestinal metaplasia.

nar epithelium shows flat cells without crypts and villi with bright intrapapillary capillary loops, while IM is characterized by uniform villiform architecture and dark goblet cells within columnar cells [9]. In a small study, the same authors reported high diagnostic accuracy and interobserver agreement of Miami classification in BE patients [12]. A recent study showed that pCLE identified patients with IM in significantly more cases than Seattle protocol (99/172 vs. $46 / 172, p<0.0001)$. Also, the difference was significant in patients with visible columnar epithelium, but not in patients without macroscopically evident columnar epithelium [13]. Therefore, pCLE can reliably distinguish the normal cardiac epithelium from IM and could be useful in patients with macroscopically present $\mathrm{BE}$ to confirm the diagnosis and in cases where IM was missed with standard biopsies by Seattle protocol.

\section{Detection of Neoplastic Lesions Related to BE}

Apart from IM, pCLE has been used to detect neoplasia associated with $\mathrm{BE}$. In practice, neoplastic lesions within $\mathrm{BE}$ are detected during the surveillance program or incidentally. On CLE exam, dysplastic/neoplastic lesions are characterized by a completely irregular "disorganized" epithelium comprising black cells, much darker than surrounding tissue with irregular leaking capillaries 


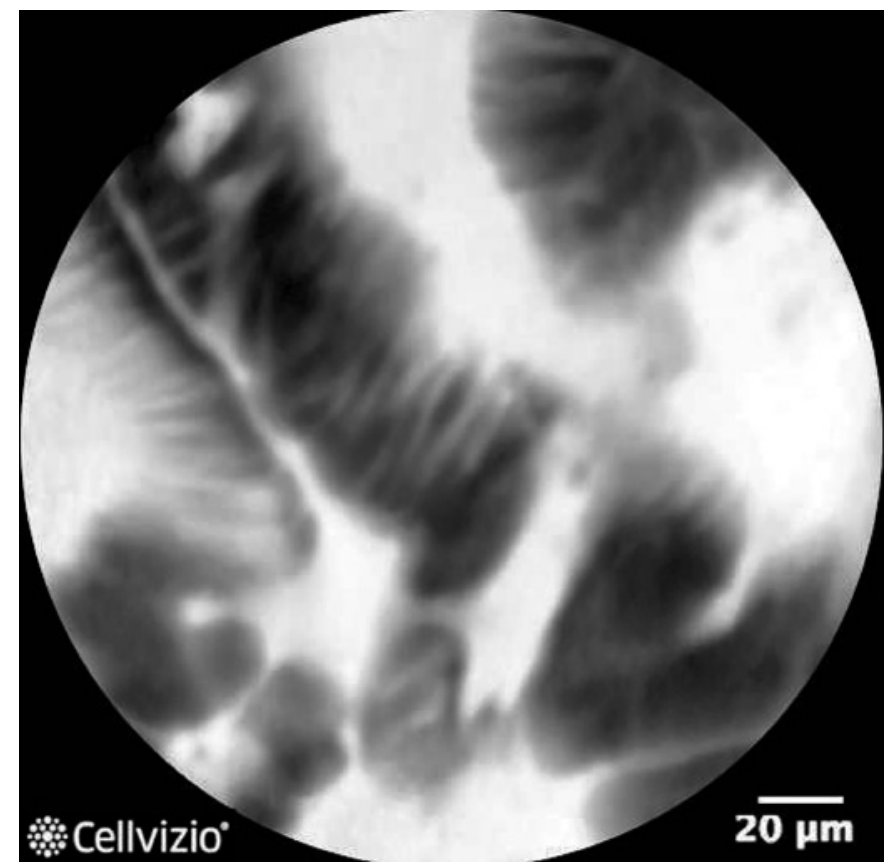

Fig. 3. Dysplasia/EAC: completely disorganized epithelium, the so-called black cells (credit: Mauna Kea Technologies). EAC, early adenocarcinoma.

in the upper and deeper mucosal layer $[1,3,11]$ (shown in Fig. 3).

Bajbouj et al. [14] performed a multicenter study in 68 patients with 670 pairs of biopsies altogether in which they compared pCLE to standard 4-quadrant biopsies to assess the possibility of pCLE to replace biopsy as standard care for BE surveillance. They showed that pCLE examination could exclude neoplasia with a specificity and NPV of 0.97 and 0.93 in blinded evaluation at least 3 months after exam and of 0.95 and 0.92 for the onsite assessment. However, sensitivity and PPV were quiet low for both settings, $28 \% / 46 \%$ in blinded evaluation and $12 \% / 18 \%$ at onsite evaluation. Thus, they concluded that pCLE is noninferior to biopsy in excluding neoplasia; however, it still cannot replace the standard biopsy regimen due to low PPV and sensitivity [14].

A study by Bertani et al. [15] aimed to compare neoplasia detection in BE between HD-WLE and PCLE in patients undergoing the $\mathrm{BE}$ surveillance program. Patients were randomized to HD-WLE alone $(N=50)$ or HD-WLE plus pCLE-targeted biopsies $(N=50)$, followed by 4 -quadrant biopsies to ensure standard care. By using pCLE, in $42 \%$ of patients, a neoplastic lesion was suspected; however, final histopathologic findings of targeted biopsies showed LGD in $24 \%$ and HGD in $4 \%$ of patients in this group. By HD-WLE only, areas suspicious for neoplasia were not observed; however, 4-quadrant biopsies proved dysplasia in $10 \%$ of patients. The overall dysplasia detection rate was $28 \%$ in the pCLE group $(p=0.04)$. Calculated sensitivity, specificity, PPV, and NPV were 100, 83,67 , and $100 \%$, respectively [15]. Another prospective, double-blind randomized study with eCLE involving 39 patients with BE revealed that eCLE-targeted biopsy led to almost doubled diagnostic yield for BE-associated neoplasia (33.7 vs. $17.2 \%$ ) and was equivalent standard care (endoscopy +4 -quadrant biopsies) for the neoplasia diagnosis. Comparing eCLE-targeted biopsies and random biopsies, there was a reduction of mucosal biopsies by $59 \%$, with comparable detection of HGD. This was observed in the high-risk group (suspected of neoplasia); however, the biopsy reduction was even greater (87\%) in low-risk patients just undergoing BE surveillance. In this surveillance group, almost two-thirds of patients did not require any biopsies because no neoplastic lesions were found during CLE. In the high-risk BE group, however, there were 2 cases of HGD detected only by random biopsies, but also 2 HGD cases detected only by eCLE, which makes neither approach completely faultless [16]. Sharma et al. [17] revealed that pCLE improves diagnostic sensitivity for detection of HGD and early adenocarcinoma (EAC) when added to HD-WLE (68.3 vs. $34.2 \%$ ), even though specificity was not significantly changed (92.7 vs. 87.8\%). Combination of NBI endoscopy and pCLE also improved sensitivity for neoplasia detection (75.8 vs. $45 \%$ ). Finally, combination of all 3 diagnostic modalities (HD-WLE, NBI, and pCLE) resulted in 100\% sensitivity for HDG and/or EAC detection which draws a conclusion that biopsies could be completely avoided in patients where the combination of the 3 modalities shows normal findings. Also, the overall number of biopsies could be significantly decreased, and diagnostic process would be facilitated [17]. Studies which assessed diagnostic accuracy, sensitivity, specificity, NPV, and PPV of pCLE in detection of BE-related neoplasia are summarized in Table 2.

In a systematic review with meta-analysis, Xiong et al. $[24,25]$ assessed accuracy of CLE (both eCLE and pCLE) for the diagnosis of HGD/EAC in BE patients. Based on study designs, they extracted data and presented their results as per-patient (PP) and per-lesion (PL) analysis. PP analysis revealed a pooled sensitivity and specificity of 89\% (95\% CI: $\left.0.82-0.94, I^{2}=31.6 \%\right)$ and $83 \%$ (95\% CI: $\left.0.78-0.86, I^{2}=90.1 \%\right)$, respectively, which indicate that CLE can reliably differentiate neoplasms from nonneoplasms in BE patients. PL analysis revealed lower pooled 
Table 2. Summary of pCLE studies in detection of HGD/EAC within BE [11, 14, 15, 17-23]

\begin{tabular}{|c|c|c|c|c|c|c|c|}
\hline Authors & Year & Pts, $n$ & DA, \% & Sens., $\%$ & Spec., \% & PPV, \% & NPV, \% \\
\hline Kiesslich et al. [11] & 2006 & 63 & 97 & 93 & 98 & 93 & 98 \\
\hline Pohl et al. [18] & 2008 & 75 & 61 & 75 & 58 & $\mathrm{n} / \mathrm{a}$ & 92 \\
\hline Bajbouj et al. [14] & 2010 & 68 & 63 & 90 & 59 & 28 & 97 \\
\hline Sharma et al. [17] & 2011 & 101 & 65 & 100 & 56 & 50 & 100 \\
\hline Bertani et al. [15] & 2012 & 50 & 86 & 100 & 83 & 67 & 100 \\
\hline Jayasekera et al. [19] & 2012 & 50 & 80 & 76 & 80 & 19 & 98 \\
\hline Legget et al. [21] & 2016 & 27 & 77 & 76 & 79 & 89 & 63 \\
\hline Shah et al. [22] & 2018 & 66 & $\mathrm{n} / \mathrm{a}$ & 67 & 98 & 67 & 98 \\
\hline Kollar et al. [23] & 2020 & 67 & 89 & 88 & 92 & 91 & 90 \\
\hline
\end{tabular}

pCLE, probe-based confocal laser endomicroscopy; HGD, high-grade dysplasia; EAC, early adenocarcinoma; BE, Barrett's esophagus; Pts, patients; DA, diagnostic accuracy; Sens., sensitivity; Spec., specificity; PPV, positive predictive value; NPV, negative predictive value.

sensitivity (77\% [95\% CI: 0.73-0.81]) and higher specificity (89\% [95\% CI: 0.87-0.90]). As mentioned earlier, CLE images and videos can be recorded and analyzed in detail after the procedure; however, true performance and real benefit from this technique should be real-time assessment and allowing real-time decision-making. The authors performed subgroup PL analysis that compared studies that assessed accuracy in real time with those where CLE diagnosis was made by blinded analysis. The results showed that blinded analysis has significantly higher specificity (94.1\% [95\% CI: 92.6-95.4] vs. $88.1 \%$ [95\% CI: 86.7-89.3]), but there was no difference noted in sensitivity (73.7\% [95\% CI: 67.5-79.3] vs. $68.3 \%$ [95\% CI: 61.8-74.3]). These data indicate that almost all real-time PL analyses were similar to overall PL analysis. However, there was significant heterogeneity between studies, some of them were not classified as high quality, and endoscopist experience bias could also affect the results [24].

Another systematic review and meta-analysis that compared NBI and CLE in diagnosis of HGD/EAC in BE patients revealed that pooled sensitivity of CLE was similar to NBI at PL level ( 72.3 vs. $62.8 \%$ and 83.8 vs. $85.3 \%$ ). Interestingly, when compared to NBI, CLE provided significant additional PL detection rate (19.3\%). Nevertheless, results of this meta-analysis should be taken with caution since significant amount of bias definitely influenced the results [25].

\section{Use of CLE with Interventional Endoscopy}

Another useful indication for pCLE might be determining the extent of dysplasia/neoplasia prior to endoscopic resection or to control the margins after the pro- cedure. Since CLE exam enables real-time assessment, it can guide the endoscopy treatment or convert initially diagnostic into therapeutic endoscopy. In 1 multicenter randomized controlled trial, Wallace et al. [26] wanted to compare, in patients that already underwent ablative therapy, whether HD-WLE alone or HD-WLE plus pCLE can guide additional ablative therapy, reducing potential undertreatment or overtreatment. When these patients returned for follow-up endoscopy, they were randomized in aforementioned groups. Then, endoscopists would make preemptive diagnosis based on HD-WLE, and in the pCLE group, they would also make pCLE diagnosis. Afterward, mucosal biopsies were taken as a referent histopathological confirmation. In cases where residual BE or neoplasia was suspected, ablative therapy was performed. Follow-up endoscopy was 3 months later, with a goal to prove if unnecessary additional ablative therapy was undertaken (suspected BE/neoplasia but negative biopsies) or if there was undertreatment (no ablation performed, but residual $\mathrm{BE} /$ neoplasia present). Unfortunately, this well-planned study was terminated after a planned interim analysis due to low conditional power that was a result of no difference between 2 groups, higher than expected residual $\mathrm{BE}$ in both groups, and high dropout rate [26]. In a case report series, patients with biopsy-proven HGD within $\mathrm{BE}$ underwent ablative therapy or resection guided by pCLE. After endoscopic therapy, pCLE exam was performed again, and 1 case showed residual dysplasia on margin which led to repeated endoscopic mucosal resection (EMR). In another case, in normal-appearing mucosa on HD-WLE, pCLE revealed residual $\mathrm{BE}$ which led to targeted biopsy that confirmed $\mathrm{BE}$ 
which was treated with radiofrequency ablation [27]. In a total of 101 patients, Park et al. [28] evaluated pCLE in the margin delineation of early gastric (not esophageal) cancer. These patients were randomized to pCLE and chromoendoscopy arms, and markings were made by electrocautery. Complete resection rate was almost the same (94.6 vs. 93.2\%). However, the median marking distance was shorter in the pCLE group although not significant ( 1.3 vs. $1.8 \mathrm{~mm}, p=0.525$ ), and the proportion of distance $<1 \mathrm{~mm}$ was higher ( 43.9 vs. $27.6 \%, p=0.023$ ). This distance difference was even greater in subgroup analysis with flat superficial lesions in the pCLE group ( 0.5 vs. 3.1 $\mathrm{mm}, p=0.007$ ) [28]. Therefore, pCLE may be used to mark the lesion more precisely prior to EMR or endoscopic mucosal dissection (ESD). However, the usefulness of this method for this indication is still questionable in the esophagus. CLE remains a promising tool for marking the lesions and providing real-time assessment and guidance of endoscopic treatment in the esophagus, but due to lack of studies and scientific evidence, standard staging based on histopathology remains the gold standard to confirm negative/positive margins.

\section{Use of CLE for Surveillance after Interventional}

Endoscopy Procedures

Regarding surveillance programs after ablation or EMR/ESD treatment of BE and associated dysplasia/neoplasia, a recent study evaluated the efficacy of pCLE versus standard biopsies in detecting persistent/recurrent IM or neoplasia. Diagnostic accuracy was $100 \%$ in the pCLE group and $94.6 \%$ for biopsies $(p=0.25)$, and sensitivity, specificity, PPV, and NPV likewise did not differ significantly [29]. Based on this study, pCLE is at least noninferior to biopsies in surveillance after endoscopic resection treatment for $\mathrm{BE}$ and related neoplasia. Nevertheless, opinion of the recent consensus report based on clinical evidence was that it needs to be further evaluated to be recommended for routine practice for this indication, despite promising results. Moreover, transformation of esophageal tissue after ablation represents an additional problem that requires defined classification for interpretation of CLE images in such patients [4].

\section{Future Perspectives of pCLE in Detection of Esophageal Neoplastic Lesions}

In forthcoming years, further pCLE technical development and innovations are to be expected. For instance, a system that has dynamic imaging depth allowing inva-

Probe-Based Confocal Laser

Endomicroscopy and Barrett's Esophagus sion depth assessment would widen the indication span. New contrast agents are also being introduced. Deoxyglucose (2-NBDG) is a new potential endomicroscopy contrast agent that exerts higher amount of fluorescence in neoplastic BE than in nonneoplastic BE. It was tested in 1 preclinical study and proved by quantitative measurement. Based on mean fluorescence intensity, neoplastic sites could be identified with a sensitivity and specificity of 96 and $90 \%$ [30].

Another possible path of endomicroscopy development is "immunoendomicroscopy." This term describes combination of CLE with basic principles of immunology. Targeting antibodies of peptides can identify desired areas of interest. An anti-EGFR antibody that binds with colonic neoplasia has been developed [31]; however, such antibody that binds with $\mathrm{BE}$ and related neoplasia is not yet available. Finally, high cost of the pCLE system still represents a significant barrier to widespread clinical implementation; therefore, low-budget, "pocket-version" systems on the market would induce the implementation of this promising method.

\section{Conclusion}

Potential clinical benefit of pCLE would be improving diagnostic accuracy for dysplasia/neoplasia detection. It offers real-time decision-making, enabling conversion of initially diagnostic endoscopy procedures into therapeutic resection procedures when needed. When combined with HD-WLE and especially with NBI, pCLE may play a significant role in the surveillance program for BE. High NPV of pCLE and especially of combination of the aforementioned diagnostic modalities offers high reliability and confidence of excluding LGD, HGD, and EAC. By principle "look more, biopsy less," pCLE offers better informed decision by where to perform tissue sampling. Therefore, it would lead to decreased number of biopsies and consequent potential complications as biopsies would be sampled "smarter." Moreover, detection of additional or residual neoplastic areas could have significant clinical impact because it may change the course of endoscopic treatment with more widespread EMR or ESD.

On the other hand, even though it has been present for over 15 years now, it still has not gained much popularity and is still being mostly used for scientific purposes. Standard surveillance guidelines recommend random tissue sampling due to simplicity of the procedure, low costs, and low risk of complications. The pCLE probe can 
inspect only a small area, and since it is not possible to take biopsies at the same time during the pCLE exam (both probe and biopsy catheter are applied through the working channel), obtaining biopsy from the exact area of pathology can be challenging and increases the risk of sampling error. Even though pCLE does increase diagnostic accuracy for detection of neoplastic transformation of $\mathrm{BE}$, at present it cannot replace standard histopathology. CLE system and examinations are still quite expensive which significantly influences the widespread use of this method. Despite promising results of pCLE in clinical studies in patients with $\mathrm{BE}$ and associated dysplastic/neoplastic lesions, its role in routine use in the esophagus remains questionable primarily due to lack of well-organized double-blind randomized trials. Low quality and heterogeneity of studies highly impacted current systematic reviews with meta-analyses as they cannot provide scientific evidence that would undoubtedly place pCLE as a useful ancillary diagnostic tool in surveillance of $\mathrm{BE}$.

\section{Acknowledgment}

The authors would like to sincerely thank Mauna Kea Technologies for providing images.

\section{Conflict of Interest Statement}

The authors have no conflicts of interest to declare.

\section{Funding Sources}

The authors did not receive any funding.

\section{Author Contributions}

Luka Vranić - concept and design of the study, literature search, draft and writing of the manuscript, table, and figure design. Tin Nadarević - corrections of the manuscript, critical revision for important intellectual concepts, table, and figure design. Davor Štimac - corrections of the manuscript, revision of the manuscript, conclusion according to data, and final approval of the version to be published.

\section{References}

1 Dunbar KB. Endomicroscopy in Barrett's esophagus. Gastrointest Endosc Clin N Am. 2013;23(3):565-79.

2 Weusten B, Bisschops R, Coron E, Dinis-Ribeiro M, Dumonceau JM, Esteban JM, et al. Endoscopic management of Barrett's esophagus: European society of gastrointestinal endoscopy (ESGE) position statement. Endoscopy. 2017;49:191-8.

3 Kollar M, Spicak J, Honsova E, Krajciova J, Vackova Z, Martinek J. Role of confocal laser endomicroscopy in patients with early esophageal neoplasia. Minerva Chir. 2018;73:41727.

4 Wang KK, Carr-Locke DL, Singh SK, Neumann $\mathrm{H}$, Bertani $\mathrm{H}$, Galmiche JP, et al. Use of probe-based confocal laser endomicroscopy (pCLE) in gastrointestinal applications. A consensus report based on clinical evidence. United European Gastroenterol J. 2015;3: 230-54.

5 ASGE Technology Committee. Confocal laser endomicroscopy. Gastrointest Endosc. 2014;80(6):928-38.

6 Wallace MB, Meining A, Canto MI, Fockens $\mathrm{P}$, Miehlke S, Roesch T, et al. The safety of intravenous fluorescein for confocal laser endomicroscopy in the gastrointestinal tract. Aliment Pharmacol Ther. 2010;31:548-52.

7 Abrams JA, Kapel RC, Lindberg GM, Saboorian MH, Genta RM, Neugut AI, et al. Adherence to biopsy guidelines for Barrett's esophagus surveillance in the community setting in the United States. Clin Gastroenterol Hepatol. 2009;7:736-10.
8 Cameron AJ, Carpenter HA. Barrett's esophagus, high-grade dysplasia, and early adenocarcinoma: a pathological study. Am J Gastroenterol. 1997;92:586-91.

9 Wallace M, Lauwers GY, Chen Y, Dekker E, Fockens P, Sharma P, et al. Miami classification for probe-based confocal laser endomicroscopy. Endoscopy. 2011;43:882-91.

10 Duits LC, Phoa KN, Curvers WL, Ten Kate FJ, Meijer GA, Seldenrijk CA, et al. Barrett's oesophagus patients with low-grade dysplasia can be accurately risk-stratified after histological review by an expert pathology panel. Gut. 2015;64:700-6.

11 Kiesslich R, Gossner L, Goetz M, Dahlmann A, Vieth M, Stolte M, et al. In vivo histology of Barrett's esophagus and associated neoplasia by confocal laser endomicroscopy. Clin Gastroenterol Hepatol. 2006;4:979-87.

12 Wallace MB, Sharma P, Lightdale C, Wolfsen $\mathrm{H}$, Coron E, Buchner A, et al. Preliminary accuracy and interobserver agreement for the detection of intraepithelial neoplasia in Barrett's esophagus with probe-based confocal laser endomicroscopy. Gastrointest Endosc. 2010;72:19-24.

13 Richardson C, Colavita P, Dunst C, Bagnato J, Billing P, Birkenhagen K, et al. Real-time diagnosis of Barrett's esophagus: a prospective, multicenter study comparing confocal laser endomicroscopy with conventional histology for the identification of intestinal metaplasia in new users. Surg Endosc. 2019; 33:1585-91.
14 Bajbouj M, Vieth M, Rösch T, Miehlke S, Becker V, Anders M, et al. Probe-based confocal laser endomicroscopy compared with standard four-quadrant biopsy for evaluation of neoplasia in Barrett's esophagus. Endoscopy. 2010;42:435-40.

15 Bertani H, Frazzoni M, Dabizzi E, Pigò F, Losi L, Manno M, et al. Improved detection of incident dysplasia by probe-based confocal laser endomicroscopy in a Barrett's esophagus surveillance program. Dig Dis Sci. 2013;58:188-93.

16 Dunbar KB, Okolo P 3rd, Montgomery E, Canto MI. Confocal laser endomicroscopy in Barrett's esophagus and endoscopically inapparent Barrett's neoplasia: a prospective, randomized, double-blind, controlled, crossover trial. Gastrointest Endosc. 2009;70:645-54.

17 Sharma P, Meining AR, Coron E, Lightdale CJ, Wolfsen HC, Bansal A, et al. Real-time increased detection of neoplastic tissue in Barrett's esophagus with probe-based confocal laser endomicroscopy: final results of an international multicenter, prospective, randomized, controlled trial. Gastrointest Endosc. 2011;74:465-72.

18 Pohl H, Rösch T, Vieth M, Koch M, Becker V, Anders M, et al. Miniprobe confocal laser microscopy for the detection of invisible neoplasia in patients with Barrett's oesophagus. Gut. 2008;57:1648-53.

19 Jayasekera C, Taylor AC, Desmond PV, Macrae $\mathrm{F}$, Williams $\mathrm{R}$. Added value of narrow band imaging and confocal laser endomicroscopy in detecting Barrett's esophagus neoplasia. Endoscopy. 2012;44:1089-95. 
20 Canto MI, Anandasabapathy S, Brugge W, Falk GW, Dunbar KB, Zhang Z, et al. Confocal endomicroscopy for Barrett's esophagus or confocal endomicroscopy for Barrett's esophagus (CEBE) trial group. In vivo endomicroscopy improves detection of Barrett's esophagus-related neoplasia: a multicenter international randomized controlled trial (with video). Gastrointest Endosc. 2014;79: 211-21.

21 Leggett CL, Gorospe EC, Chan DK, Muppa P, Owens V, Smyrk TC, et al. Comparative diagnostic performance of volumetric laser endomicroscopy and confocal laser endomicroscopy in the detection of dysplasia associated with Barrett's esophagus. Gastrointest Endosc. 2016;83:880-e2.

22 Shah T, Lippman R, Kohli D, Mutha P, Solomon S, Zfass A. Accuracy of probe-based confocal laser endomicroscopy (pCLE) compared to random biopsies during endoscopic surveillance of Barrett's esophagus. Endosc Int Open. 2018;6:E414-20.

23 Kollar M, Krajciova J, Prefertusova L, Sticova E, Maluskova J, Vackova Z, et al. Probe-based confocal laser endomicroscopy versus biop- sies in the diagnostics of oesophageal and gastric lesions: a prospective, pathologist-blinded study. United European Gastroenterol J. 2020;8:436-43.

24 Xiong YQ, Ma SJ, Zhou JH, Zhong XS, Chen Q. A meta-analysis of confocal laser endomicroscopy for the detection of neoplasia in patients with Barrett's esophagus. J Gastroenterol Hepatol. 2016;31:1102-10.

25 Xiong YQ, Ma SJ, Hu HY, Ge J, Zhou LZ, Huo ST, et al. Comparison of narrow-band imaging and confocal laser endomicroscopy for the detection of neoplasia in Barrett's esophagus: a meta-analysis. Clin Res Hepatol Gastroenterol. 2018;42:31-9.

26 Wallace MB, Crook JE, Saunders M, Lovat L, Coron E, Waxman I, et al. Multicenter, randomized, controlled trial of confocal laser endomicroscopy assessment of residual metaplasia after mucosal ablation or resection of GI neoplasia in Barrett's esophagus. Gastrointest Endosc. 2012;76:539-e1.

27 Johnson EA, De Lee R, Agni R, Pfau P, Reichelderfer M, Gopal DV, et al. Probebased confocal laser endomicroscopy to guide real-time endoscopic therapy in Barrett's esophagus with dysplasia. Case Rep Gastroenterol. 2012;6:285-92.

28 Park JC, Park Y, Kim HK, Jo JH, Park CH, Kim $\mathrm{EH}$, et al. Probe-based confocal laser endomicroscopy in the margin delineation of early gastric cancer for endoscopic submucosal dissection. J Gastroenterol Hepatol. 2017;32:1046-54.

29 Krajciova J, Kollar M, Maluskova J, Janicko M, Vackova Z, Spicak J, et al. Confocal laser endomicroscopy versus biopsies in the assessment of persistent or recurrent intestinal metaplasia/neoplasia after endoscopic treatment of Barrett's esophagus related neoplasia. J Gastrointestin Liver Dis. 2020;29:305-12.

30 Thekkek N, Maru DM, Polydorides AD, Bhutani MS, Anandasabapathy S, Richards-Kortum R. Pre-clinical evaluation of fluorescent deoxyglucose as a topical contrast agent for the detection of Barrett's-associated neoplasia during confocal imaging. Technol Cancer Res Treat. 2011;10:431-41.

31 Liu J, Zuo X, Li C, Yu T, Gu X, Zhou C, et al. In vivo molecular imaging of epidermal growth factor receptor in patients with colorectal neoplasia using confocal laser endomicroscopy. Cancer Lett. 2013;330:200-7. 